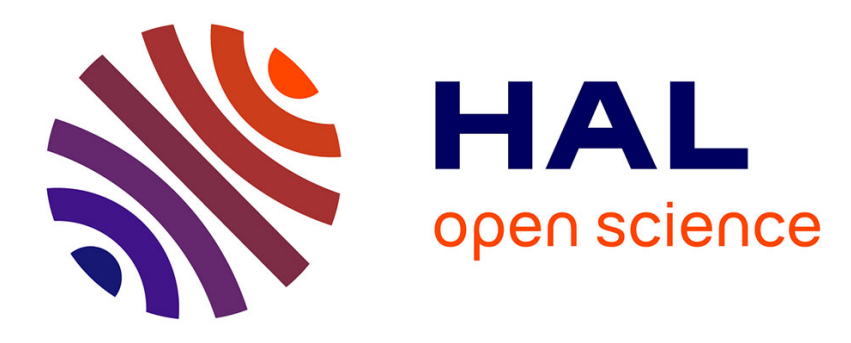

\title{
Microstructures, textures and mechanical properties after large strain
}

N. Hansen, T. Leffers

\section{To cite this version:}

N. Hansen, T. Leffers. Microstructures, textures and mechanical properties after large strain. Revue de

Physique Appliquée, 1988, 23 (4), pp.519-531. 10.1051/rphysap:01988002304051900 . jpa-00245799

\section{HAL Id: jpa-00245799 \\ https://hal.science/jpa-00245799}

Submitted on 1 Jan 1988

HAL is a multi-disciplinary open access archive for the deposit and dissemination of scientific research documents, whether they are published or not. The documents may come from teaching and research institutions in France or abroad, or from public or private research centers.
L'archive ouverte pluridisciplinaire HAL, est destinée au dépôt et à la diffusion de documents scientifiques de niveau recherche, publiés ou non, émanant des établissements d'enseignement et de recherche français ou étrangers, des laboratoires publics ou privés. 


\title{
Microstructures, textures and mechanical properties after large strain
}

\author{
N. Hansen and T. Leffers
}

\author{
Metallurgy Department, Ris $\varnothing$ National Laboratory, 4000 Roskilde, Denmark
}

(Reçu le 26 mai 198\%, accepté le 28 septembre 1987)

\begin{abstract}
Résumé.- On passe en revue les propriétés mécaniques, la texture et la microstructure des matériaux polycristallins. Les modèles existants pour la déformation sont discutés et les propriétés mécaniques sont corrélées avec la microstructure. Enfin, l'anisotropie et les relations constitutives sont discutées.
\end{abstract}

\begin{abstract}
Microstructure, texture and mechanical properties of polycrystalline materials after medium and large strains are reviewed. The existing deformation models are discussed on the basis of the evidence presented, and mechanical properties and microstructure are correlated. Finally,
\end{abstract} anisotropy and constitutive relations are discussed briefly.

CONTENTS

1. Introduction

2. Deformation microstructures

2.1. Dislocation cells

2.2. Bands

2.3. Surface observations

2.4. Effect of metallurgical parameters

3. Deformation textures

3.1. Rolling textures

3.2. Effect of metallurgical parameters

4. Deformation models

4.1. Models and microscopical observations

4.2. Models and texture

4.3. Improved models

5. Mechanical properties

5.1. Flow stress-strain behaviour

5.2. Flow stress-texture ( $M$ factors)

5.3. Flow stress-microstructural evolution

5.4. Flow stress-cell size

5.5. Effect of metallurgical parameters

5.6. Anisotropy

6. Constitutive relations

Concluding remarks

\section{INTRODUCTION}

Deformation of metals and alloys is accompanied by changes in microstructure and texture. These changes can provide information about the mechanisms of plastic flow and about strain-induced changes in the mechanical properties. The present paper will deal with medium and large deformations of polycrystalline materials at low temperatures (where climb can be neglected). A characteristic behaviour is examplified by f.c.c. metals (aluminium and copper), and it is discussed how this behaviour is modified by metallurgical parameters such as grain size and volume fraction and size of hard particles. The microstructural and textural information is related to the flow stress-strain behaviour, and the relationship between the flow stress and various microstructural parameters is described. Plastic anisotropy is described briefly, and finally the possibility of developing constitutive relations on theoretical basis is discussed.

\section{DEFORMATION MICROSTRUCTURES}

The dislocation microstructures in deformed materials have been studied extensively in order to understand the deformation mechanisms and to derive relations between the flow stress and microstructural parameters. In general the dislocation structures develop with increasing strain from tangled dislocations to well-defined dislocation arrays forming a regular cell structure. Superimposed on the cell structure, a banded dislocation structure is frequently observed. The various types of dislocation arrangements will be summarized in the following. In section 4 these microscopical observations (combined with texture observations) will be discussed in relation to the existing deformation models.

2.1. Dislocation cells.

The formation of dislocation cells has been discussed on the basis of different mechanisms of which the energy minimization principle recently has been treated in detail [1]. It follows from this principle that a uniform or statistical distribution of dislocations is unstable since the dislocation energy can be reduced when the dislocations cluster into stress-screening arrays. The degree of this reduction depends on the availability of slip systems because an increase in the number of Burgers vectors permits the formation of arrays of lower energy. Mobility of dislocations is another important factor, which may be influenced 
by cross slip. Clustering of dislocations leads to a structure consisting of volume elements almost free of dislocations separated by densely packed dislocation arrays, and this is what one observes at medium and high strains. As an example figure 1 shows the cell structure of aluminium cold drawn 80 $\%$.

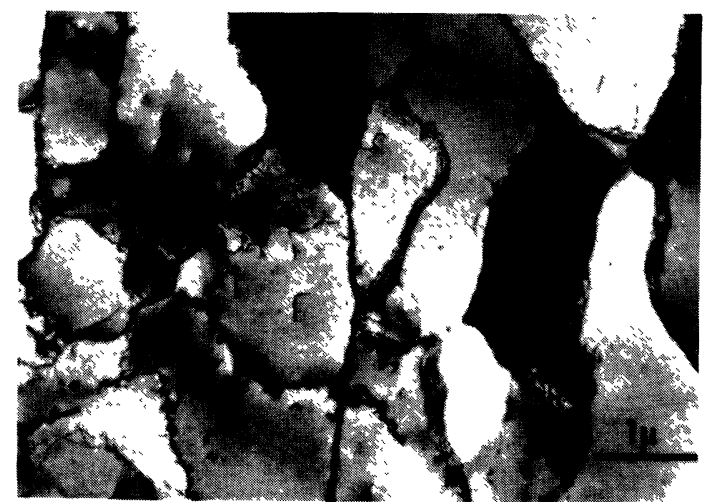

Fig. 1. TEM micrograph of $80 \%$ cold-drawn 99.998\% Al, longitudinal section. From [2].

An alternative mechanism for the formation of cell structures has been suggested [3] on the basis of the existence of 'hard areas' in the crystals which are impenetrable for dislocations. Three-dimensional boundaries may then form and there will be a strong tendency that dislocation are placed on each side of the boundary according to their sign. Thereby a bipolar wall is formed, which contains a high local internal stress. This is in contrast to the virtually stress free walls formed according to the energy minimization principle.

For a regular cell structure as shown in Fig. 1 characteristic features are the cell size and the misorientation across the cell walls. The dislocation arrangement in the cell walls may be quite simple as in tilt and twist boundaries. However, in most cases especially at medium and large strains the arrangements are far more complex, and large variations between the walls are observed. It is a general observation that the cell size and the misorientation across the cell walls depend on the plastic strain, and it is frequently observed that there is a decrease in cell size together with an increase in misorientation when the strain is increased $[4,5,6]$. Figure 2 illustrates the cell size-strain dependence for aluminium deformed by drawing, and it is noted that the cell size decreases continuously with increasing strain. This behaviour is typical when the deformation mode is drawing or rolling [4], whereas, for material deformed in tension, the cell size may reach a constant value at a strain of the order of 0.1-0.2 $[5,6]$. An increase in strain above this values leads to an increase in the misorientation across the cell walls indicating an increased dislocation density in the walls [6]. This effect of the deformation mode is at present unexplained and leads into the general problem as to how the dislocations are arranged in the cell walls. Studies of such

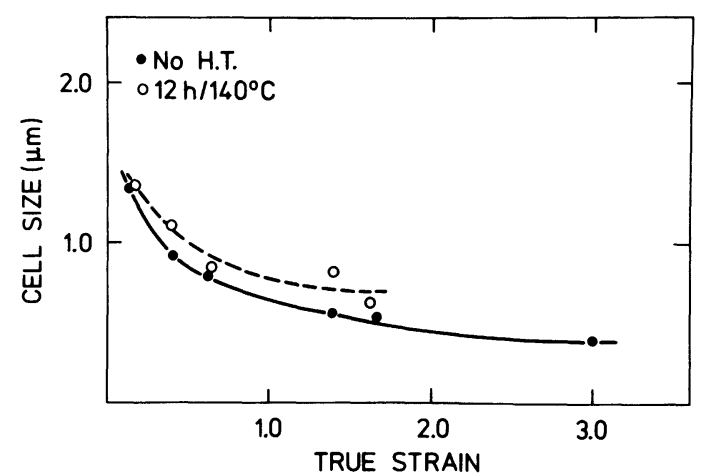

Fig. 2. Cell size as a function of strain by cold drawing of $99.998 \%$ Al (from [2]). The material has been examined as-deformed and after a heat treatment at $140^{\circ} \mathrm{C}$ for 1 hour.

structures are quite difficult but modern microscopical and non-microscopical [7] techniques of fer new possibilities.

2.2. Bands.

The regular cell structure may contain a number of special features often in the shape of bands. One characteristic feature is 'dense dislocation walls' (DDWs) [8]. These are structures running continuously and rather straight for distances of tens or a few hundreds of micrometers and aligned close to active slip planes. Their width and spacing are of the order of $0.2 \mu \mathrm{m}$ and $2-4 \mu \mathrm{m}$, respectively. They are narrow walls incorporating a greater misorientation than ordinary cells (up to $4^{\circ}$ ). In figure 3 DDWs delineate the abrupt contrast changes between sequences of similarly oriented cells from lower left to upper right. The material in figure 3 is polycrystalline aluminium, but DDWs have also been observed in aluminium single crystals [9] as well as in polycrystalline copper [10] and nickel [11]. On the basis of those findings it has been suggested [12] that the DDWs are the counterpart of tilt walls parallel to the primary slip planes (mats) as observed in deformed single crystals.

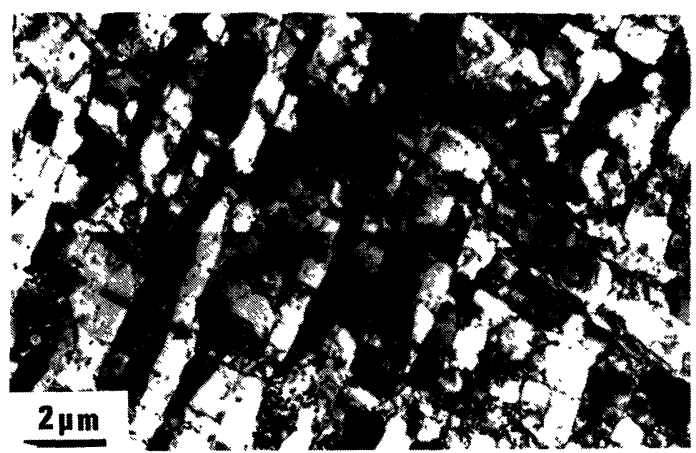

Fig. 3. TEM micrograph of $15 \%$ cold-rolled AI 0.6 vol.\% $\mathrm{Al}_{2} \mathrm{O}_{3}$ (by courtesy of $\mathrm{B}$. Bay). DDWs delineate the abrupt contrast changes between similarly oriented cells stretching from lower left to upper right. A microband is marked with an arrow. 


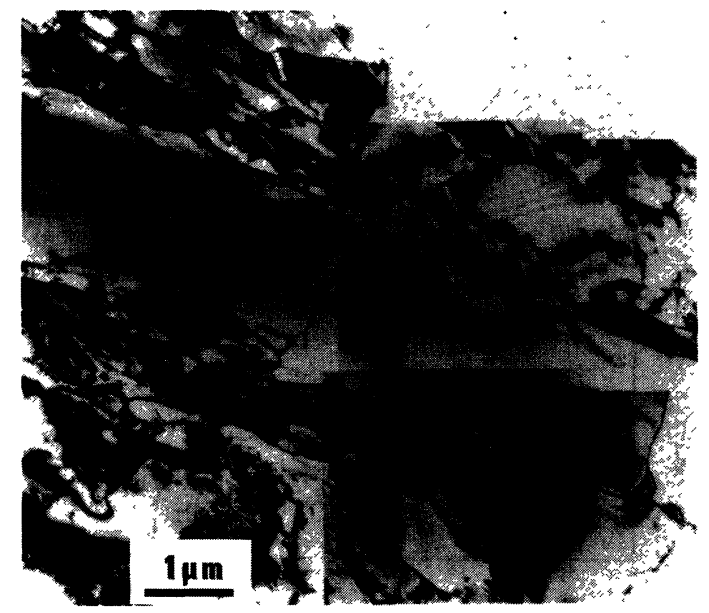

Fig. 4. TEM micrograph of $15 \%$ cold-rolled 99.998\% Al showing the dislocation arrangement in two microbands (by courtesy of B. Bay).

Superimposed on the regular dislocation cell structure 'microbands' composed of much smaller cells are often observed in metals such as aluminium [13] and copper [14]. In general such bands contain a relatively high dislocation density, their width is a few tenths of a micrometer and they extend typically from tens to hundreds of micrometers. These bands are almost parallel to active slip planes, their spacing is typically 2-5 $\mu \mathrm{m}$ and the misorientation of the bands with respect to the surrcunding matrix is typically less than $2^{\circ}$. In $\mathrm{Al}-\mathrm{Mg}$ far higher misorientations have been reported for local positions along microbands [15]. Examples of microbands in aluminium can be seen in figures 3 and 4 . The microstructure of microbands in copper is more regular [14]. Possible mechanisms for the formation of microbands and their role in the deformation process have been discussed in refs. $[9,14,16]$ for instance.

The bands discussed above are related to the orientation of the slip systems in the grains in which they are found. In contrast, the orientation of shear bands does not correspond to the active slip systems and they are not limited by the grain boundaries. The characterization of shear bands and the mechanisms behind their formation will not be covered in the present paper.

In contrast to the bands described so far, other types of 'bands' e.g. deformation bands, transition bands and grain boundary bands incorporate larger misorientations across them (tens of degrees) and extend over relatively large volumes of materials $[4,17]$. These bands start to form at relatively small strains and their number and the misorientation across them increases when the strain is increased. The occurrence of such bands reflects that grains can separate into regions deforming on different slip systems or different combinations of slip systems or by various degrees of slip on a single set of slip systems. It appears that not only the lattice orientation but also the strain is subjected to local variations.

\subsection{Surface observations.}

Information complementary to that obtained in the studies of bulk dislocation structures can be obtained from slip line observations on the surface. In pure f.c.c. metals slip line patterns include an elementary structure more or less uniformly distributed over the surface consisting of fine lines with a spacing of the order of 20-40 nm which is normally only observed at low strains and a coarser structure of stronger slip lines which in medium and high stacking fault energy materials may cluster to form very coarse slip bands of variable spacing. The slip bands represent rather large shears (offset typically 1000 Burgers vectors or more). The distribution of slip lines/bands reflect the fragmentation described in 2.2. In the following some recent observations are presented.

A direct comparison of surface and bulk structures is possible by examining thin foils from a surface [8]. The structures obtained by this technique can be examined using a high voltage electron microscope. The results show that the dislocation structure reveals the direction of the active slip planes as indicated by the slip bands on the surface. Furthermore, DDWs and microbands appear to be individually associated with the corresponding slip bands. These observations indicate that the DDWs outline the large initial cells formed during primary slip and that slip in a cell structure occurs over distances which may involve a number of cells. This latter suggestion is in accord with what is often observed in in situ straining experiments in a high voltage electron microscope, namely that dislocations stop at cell walls but then may continue after momentary arrest [18].

\subsection{Effect of metallurgical parameters.}

Metallurgical parameters as grain size, second phase particles, elements in solid solution etc. affect the slip patterns and the resultant microstructures. Most of these effects have not been quantified, except for the cell size, which tends to decrease when solute elements and particles are introduced. Furthermore, the cell size is affected by the stacking fault energy of the base metal [5]. The effect of grain size and large and small particles on the slip pattern will be discussed in the following.

a) Grain size. Surface relief patterns show that the slip line structure is finer in fine-grained specimens for small degrees of deformation. The slip pattern is inhomogeneous in nature. However, the non-uniform distribution of slip bands tends to be least pronounced in fine-grained specimens [19]. With increasing degree of deformation the slip pattern becomes quite similar in fine-grained and coarse-grained specimens [19].

Dislocation structures examined by. TEM are inhomogeneous and the grain size appears to have relatively little effect on the structures observed, but it is generally found that the dislocation density at a constant strain tends to increase when the grain size is decreased [17]. Grain fragmentation as studied by selected area channeling patterns appears to be less pronounced in fine-grained 
than in coarse-grained specimens (J.B. Bilde-Sørensen, private communication).

b) Large particles. The presence of coarse hard particles ( $0.1 \mu \mathrm{m}$ ) causes plastic inhomogeneity at the interface between particles and matrix which can lead to the formation of local deformation zones where the lattice is rotated significantly with respect to the matrix [20]. The orientation spread within the complete deformation zone has been determined by microtexture measurements for single crystals [21] and it has been found that parts of the deformation zone at the particles have misorientations up to $40^{\circ}$, but a relative large fraction of the deformation zone has orientations very similar to that of the surrounding matrix. Systematic studies have not been carried out on polycrystalline materials but a number of findings indicate that the effect of particles is similar to that observed for single crystals. However, it has also been-observed that rotations even at relatively large particles may be small [22]. Furthermore, it has been observed that particles agglomerated in groups and particles present in transition bands and at grain boundaries may lead to enhanced rotations and increased density of dislocations.

c) Small particles. Small hard (non-shearable) particles $(<0.1 \mu \mathrm{m})$ refine the slip pattern, and a general finding by SEM is that the slip lines are fainter, more closely spaced and often interrupted when compared with material without particles [23]. The dislocation structures in the bulk are also affected by the presence of particles, which leads to higher dislocation density and a smaller subgrain size at a given strain [2]. The formation of structural features indicating lattice rotations such as transition bands and grain boundary bands have been studied in $\mathrm{Al}-\mathrm{Al}_{2} \mathrm{O}_{3}$ materials [23]. The general observation is that the ef fect of particles is small except at low degrees of deformation, where the formation of transition bands is more pronounced in aluminium without particles. This finding seems to reflect a homogenizing effect of particles on the deformation pattern.

\section{DEFORMATION TEXTURES}

The deformation textures of f.c.c. materials have been studied extensively, particularly the rolling textures. Such studies combined with model calculations can give important information about deformation mechanisms and about the ef fect of texture on the relationship between the flow stress and strain and on anisotropy. The emphasis in the present work will be on materials developing a coppertype rolling texture, in particular aluminium.

3.1. Rolling textures.

Figure 5 shows the texture of pure aluminium (99.998\%) rolled $90 \%$ as expressed in the $\{111\}$ pole figure. The aluminium texture is a coppertype texture (as opposed to the brass-type texture developed in materials with low stacking-fault energy). For comparison with figure 5 the $\{111\}$ pole figure for rolled copper is shown in figure 6 . These figures show that the textures in aluminium

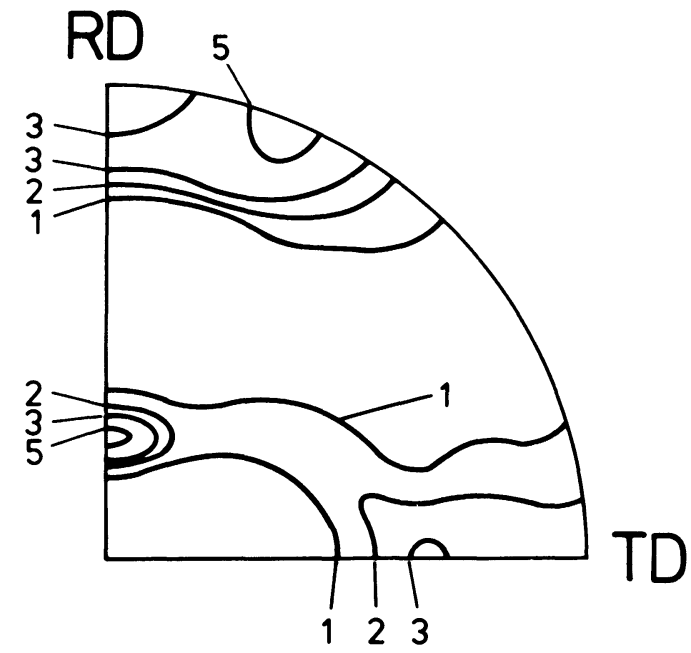

Fig. 5. \{111\} pole figure for pure aluminium rolled to $90 \%$ reduction (by courtesy of D. Juul Jensen).

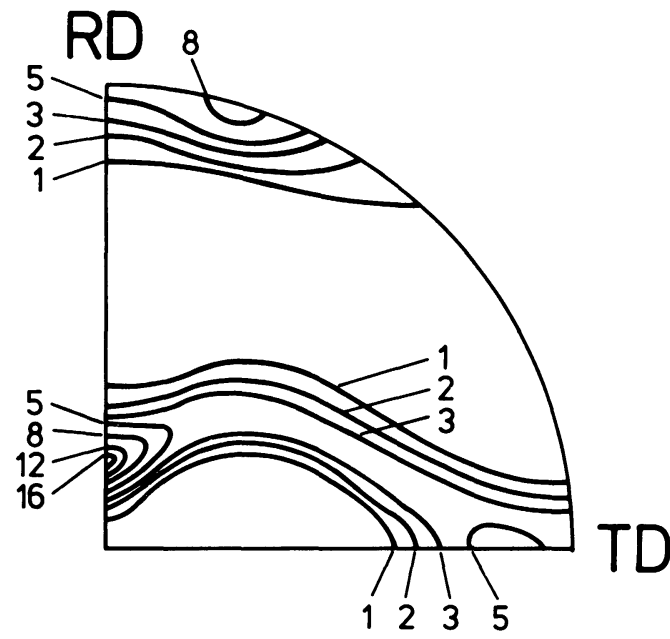

Fig. 6. \{111\} pole figure for OFHC copper rolled to $95 \%$ reduction.

and copper are comparable, although not identical. The differences can be elucidated by comparing the experimental pole figures with those calculated on the basis of texture models. The Taylor model [25] and the relaxed-constraint model $[26,27,28]$ produce the pole figures shown in figures 7 and 8 [29]. A comparison of figures $5,6,7$ and 8 shows that the texture of copper at high strains is very similar to the texture calculated by the relaxedconstraint model, whereas the texture of aluminium is somewhere between the relaxed-constraint and the full-constraint texture. The same trend, i.e. that the texture is in between a relaxed-constraint and a full-constraint texture, is also observed in the pole figures for commercially pure aluminium quoted in the literature (e.g. [24]), but normally these pole figures come closer to relaxed constraint than that in fig. 5. It should be mentioned that textures very similar to the full-constraint texture in figure 7 have been observed experimen- 


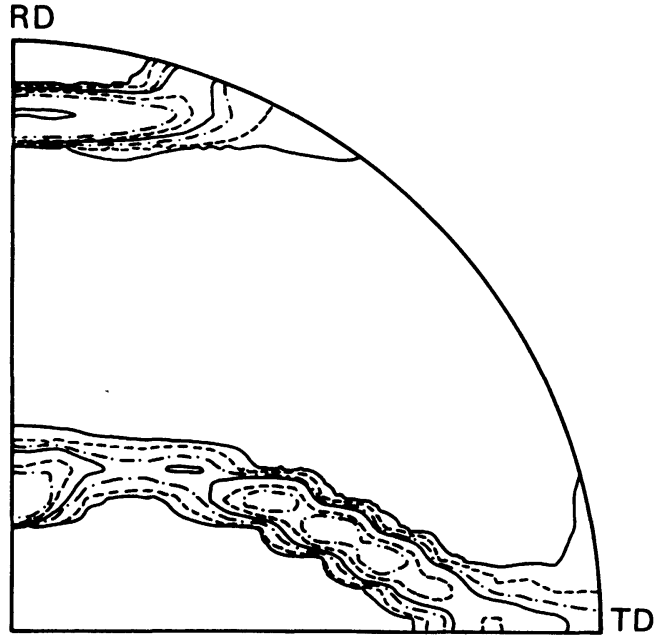

Fig. 7. \{111\} pole figure for a simulated fullconstraint Taylor texture (from [29]). Contour lines for pole densities in the range 0.5-19.

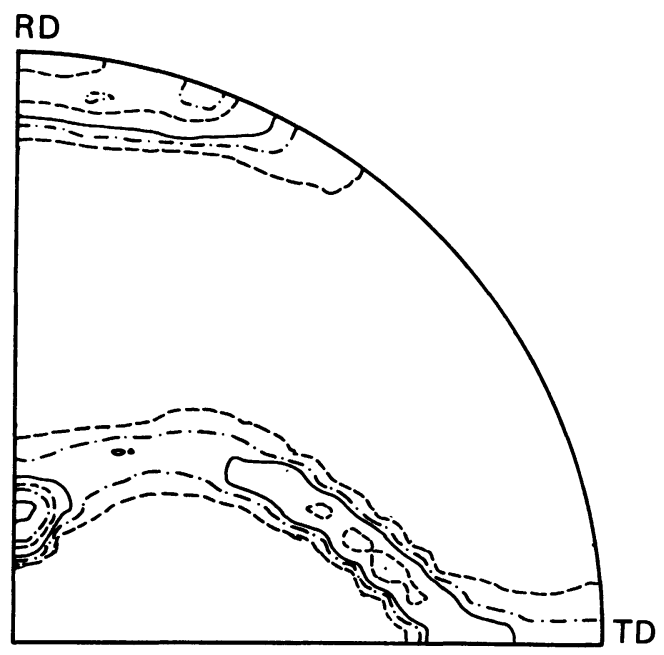

Fig. 8. \{111\} pole figure for a simulated relaxed-constraint texture (from [29]). Contour lines for pole densities in the range 1-13.

tally for special conditions, viz. rolling of aluminium (and aluminium alloys) at elevated temperature $[24,30]$. The texture of copper at lower rolling reduction is, like the fully developed aluminium texture, somewhere between relaxed constraint and full constraint, but closest to relaxed constraint [29].

3.2. Effect of metallurgical parameters.

Deformation textures are qualitatively and quantitatively affected by a number of metallurgical parameters including the stacking fault energy. However, in the following we shall concentrate on the effect of grain size and dispersed particles (as in the section on microstructures) and hence neglect the problem of the formation of the brass- type texture (e.g. $[31,32])$.

a) Effect of grain size. The effect of grain size on the textural development during cold rolling has been studied in some detail $[19,33]$, and 1 is found that an increase in grain size reduces the rate of textural development and decreases the maximum orientation densities even at large degrees of deformation (figure 9). In studying the effect of grain size, an inherent problem is that a variation in this parameter normally is accompanied by a change in the initial texture. Thus the textural development observed might be a combined effect of the two parameters. This has been taken into account in an experimental investigation of the effect of grain size on the texture and microstructure of commercially pure aluminium (approx. 99.5\%) cold rolled from 15 to $95 \%$ reduction in thickness at room temperature [34] and in an investigation by computer simulation [29]. The results from these studies show that both the initial grain size and the starting texture have pronounced effects on the development of the rolling texture and that these effects are significantly reduced at large rolling reductions.

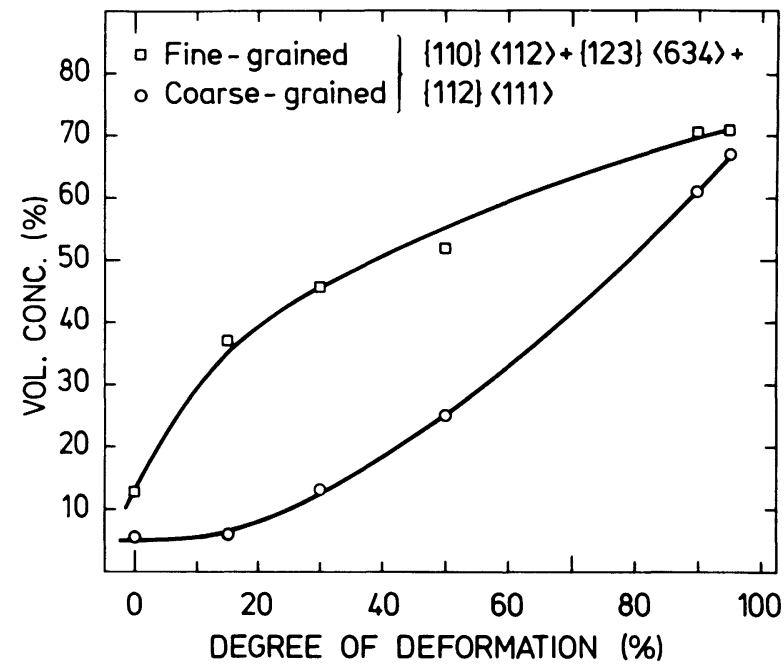

Fig. 9. The development of rolling texture in fine-grained $(50 \mu \mathrm{m})$ and coarse-grained $(300$ $\mu \mathrm{m})$ aluminium (from [19]). The rolling texture is characterized by the sum of the orientations within $15^{\circ}$ from $\{110\}\langle 112\rangle,\{123\}\langle 634\rangle$ and \{112\} 〈111〉 in ODF space.

b) Effect of large particles. The ef fect of coarse hard particles $(>0.1 \mu \mathrm{m})$ on the deformation texture has not been investigated systematically. However, a number of experimental findings for various materials indicates that the presence of large particles in general causes a weakening of the texture and that this effect becomes more pronounced when the volume concentration of particles is large [34].

The randomizing effect of large particles on the textural development can be related to the for- 
mation of deformation zones at the particles where the lattice is rotated significantly with respect to the matrix. How much the deformation texture in a polycrystaline material actually is weakened by the presence of large particles will therefore depend on the volume fraction of particles, the size of the deformation zones and the lattice rotations within the zones. But besides that, the spread of the texture in the particle free matrix is important [34]. This spread may depend on parameters such as material, starting texture, grain size, degree of reduction etc. To determine the weakening of the deformation texture the lattice rotations in the deformation zones around the particles have to be compared with the spread of the matrix texture away from the skeleton line of the orientation tube which may be $5-10^{\circ}$, in some cases up to $15^{\circ}$. If the maximum lattice rotations in the deformation zones are small $\left(5-15^{\circ}\right)$ and therefore comparable to the spread of the matrix texture, most of the orientations in the deformation zones will be present in the matrix texture and the texture will only be slightly weakened. This would be the case in lightly deformed materials containing relative small particles. However, at medium and large degrees of deformation the lattice rotations in the deformation zones can be much larger than the spread of the matrix texture, e.g. 30-40 and the texture will be significantly weakened [34] .

c) Effect of small particles. The effect of small hard (non-shearable) particles $(<0.1 \mu \mathrm{m})$ on the deformation textures have been investigated in a number of studies (see [34]), but it is difficult to establish a trend. For small volume concentration of particles some studies have shown that the texture is strengthened whereas in other cases a neutral or a weakening effect has been observed. For large volume concentrations of particles, the general effect appears to be that the deformation texture is weakened.

One might imagine that homogenization of the slip pattern is the predominant effect for small volume fractions of particles and that this might produce texture strengthening. For large fractions the distortion of the slip pattern would dominate, resulting in a weakening of the texture.

\section{DEFORMATION MODELS}

In this section the existing models for the plastic deformation of polycrystals will be discussed in terms of the experimental observations of microstructure and texture reviewed in sections 2 and 3 . Only the observations on single-phase materials will be dealt with. The knowledge of the effects of particles is so limited that any discussion of their model implications beyond the remarks already made in sections 2 and 3 would be premature. First the spectrum of existing models will be briefly reviewed.

The Taylor model [25] describes a homogeneous slip pattern with basically no qualitative difference between the different slip systems, only a quanti- tative difference between the amount of shear. The relaxed-constraint model (e.g. [26,27]) relies on heterogeneous plastic interaction between the grains to account for a minor part of material continuity, but the major fraction of slip is homogeneous. As in the Taylor model there is only a quantitative difference between the different slip systems. The relaxation of constraints is linked to the shape of the grains at large strain (there is no relaxation for equiaxed grains). Relaxation of constraint has been suggested earlier with different arguments [28]. Other models, like the 'modified Sachs model' (e.g. [35]), rely on plastic interaction between the grains (manifesting itself in a heterogeneous slip pattern, e.g. $[35,36])$ to account for a large part of material continuity. In the modified Sachs model there is a qualitative difference between the 'primary' slip systems (the slip systems with the highest resolved shear stress from the applied stress) and the other systems.

Formally, the plastic interaction between the grains is inversely related to the interaction with a continuum matrix. In the Taylor model the individual grains have a very strong (rigid-plastic) interaction with the continuum matrix and hence no plastic interaction with the actual neighbours. The more the formal interaction with the continuum matrix is relaxed (e.g. by relaxation of individual constraints), the more the models rely on plastic interaction between the grains for strain accommodation. The self-consistent scheme of Berveiller and Zaoui and co-workers (e.g. [37]) covers the whole range from (elastic-plastic) Taylor-type deformation to deformation with strong plastic interaction by adjusting the strength of the interaction with the continuum matrix. Even when plastic grain interaction is implied in a model, this does not necessarily mean that the corresponding slip is accounted for when the model is used for quantitative simulation (texture simulation for instance).

4.1. Models and microscopical observations.

In the Taylor model the number of active slip systems required and accounted for is 5 (on 3 or 4 slip planes in f.c.c. materials). In the relaxedconstraint model the number of slip systems formally accounted for is marginally smaller, and in models like the modified Sachs model it is substantially smaller. But when the slip systems not formally accounted for are added, the total number of slip systems would, in all models, come to at least 5 as in the Taylor model. Thus, the total number of slip systems is not the major issue in the microscopical investigation of the slip pattern. The important points are the homogeneity or heterogeneity of the distribution of slip and the character of the different slip systems.

The slip pattern varies throughout the average grain - as it seems more so in coarse-grained than in fine-grained materials. This is not basically incompatible with the Taylor model because of the ambiguity in the selection of slip systems, but it does support models like the relaxed-constraint model or the modified Sachs model which positively include heterogeneous deformation. Furthermore, the 
indication is that the heterogeneous slip pattern is accompanied by heterogeneous strain which is incompatible with the Taylor model. The effect of grain size on the slip heterogeneity is also difficult to accommodate within the Taylor model.

The DDWs and the microbands in the bulk structure and the corresponding bands with very intense slip on the surface seem to reflect a very high concentration of slip. In a specific area one normally observes one or at the most two systems of DDWs and microbands, reflecting a morphological difference between some 'primary' slip systems and the other systems. This would agree with the assumption in the modified Sachs model that there is a basic difference between the different slip systems. Recent observations [15] demonstrate activation of localized slip in the neighbouring grain where a microband ends at a grain boundary as also assumed in the modified Sachs model.

In conclusion the microscopical observations support the models which include heterogeneous deformation and plastic interaction between the grains; some observations indicate that heterogeneity and interaction have a major role to play. A substantial effort is still required to make the observations more conclusive, but it is clear that microscopical observations of slip morphology is a very important supplement to investigations which provide macroscopic or statistical information about the slip processes like texture investigations.

4.2. Models and texture.

As shown in section 3.1. the experimental rolling texture in materials with medium and high stacking fault energy are apparently in quite good agreement with predictions based on the relaxed-constraint model, However, a more detailed comparison of experiments and predictions reveals some discrepancies.

A comparison of figures 6 and 8 illustrates that the texture of copper after heavy rolling is quite close to the texture simulated with the relaxedconstraint model. The constraint relaxed in figure 8 is that corresponding to the component of change in shape which is most easily accommodated by plastic interaction between the strongly elongated and flattened grains (the relaxation of constraint follows the 'lath mode' [38]). However, there is another constraint which, judged from the shape of the heavily rolled grains, would be almost as likely to be relaxed (the two constraints relaxed simultaneously would correspond to the 'pancake mode' [38]). Simulation according to the pancake mode produces a texture which is clearly less similar to the copper texture than the texture simulated according to the lath mode [38], i.e. relaxation of constraints as derived from the grain shape is not without problems.

The texture of copper after moderate degrees of reduction (e.g. 40\%) is close to relaxed constraint, whereas grain-shape considerations would lead to the use of full constraint.

Compared with copper the texture in heavily rolled aluminium has shifted somewhat from a relaxedconstraint (lath) texture towards a full-constraint Taylor texture, which has no obvious explanation in terms of the grain shape.

There is some ambiguity in the selection of slip systems in the Taylor model and also a (smaller) ambiguity in the relaxed-constraint model, which gives some ambiguity in the simulated textures. But it is unlikely that the above problems with the copper and the aluminium textures can be explained as variations within this range of ambiguity; the actual ambiguity in the simulated rolling textures is quite small [39]. The implication is that the relaxed-constraint model, in particular the grainshape argument for relaxation of constraint, needs to be reconsidered.

4.3. Improved models.

There is little doubt that plastic interaction between the grains does play a significant role. The development of rational schemes which formally include grain interaction is going to be an obvious focal point in future modelling work. The ideal way of doing this would be to include the actual interaction between specific grains (rather than dealing with individual grains which interact more or less with a continuum matrix). The improved model(s) should also cover the effect of grain size (increasing slip heterogeneity and decreasing rate of texture development with increasing grain size). Another line to persue is the investigation of rate dependent models (e.g. [40]), even though it is an open question whether viscoplastic flow as implied in such models is relevant at low temperature.

With a view on the history of modelling it might be relevant to underline the extreme importance of a close connection between modelling work and experimental observations. The use of experimental textures as starting textures in modelling can facilitate comparison with experimental textures and widen the experimental basis for modelling [29].

\section{MECHANICAL PROPERTIES}

The effect of cold deformation on the mechanical properties is mainly to increase the flow stress, decrease the ductility and to introduce anisotropy. In the present section we shall concentrate on the flow stress-strain behaviour and discuss the related changes in microstructure and texture. The relationship between the flow stress and various microstructural parameters will also be described. Finally the anisotropy of the mechanical properties will be discussed; in this connection anisotropy refers specifically to the difference in properties in different directions. But of course even the flow stress in one direction in a textured material is an anisotropic property since it depends on the direction chosen.

5.1. Flow stress-strain behaviour.

In tension it is generally observed that the work hardening rate changes with the strain from constancy (stage II), through an interval with decreasing rate (stage III) to a range with constant 
rate (stage IV). Only at very high strains a saturation of the work hardening rate has been observed. As an illustration the flow stress-strain curves of cold-rolled and of wire-drawn aluminium are shown in figure 10.

Flow softening has for instance been observed in copper (99.9999\%) when measuring the flow stress $(0.2 \%$ of fset) of specimens cold-rolled to a strain above approximately 1.6 [42]. Flow softening was not observed for copper of the same purity tested in tension [43], whereas it was found in compression at strains above approximately 1.1 [43]. This is illustrated in figures 11 and 12 .

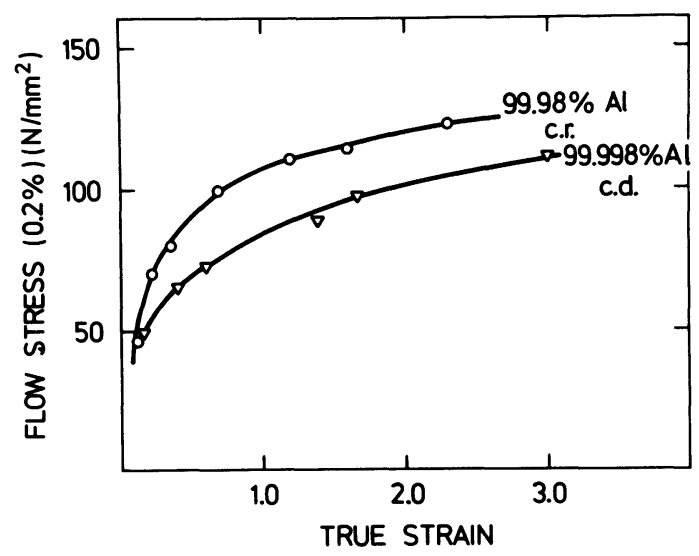

Fig. 10. Flow stress-strain curves $(0.2 \%$ of $f-$ set) for aluminium reduced by cold-rolling (c.r.) [41] and cold-drawing (c.d.) [2].

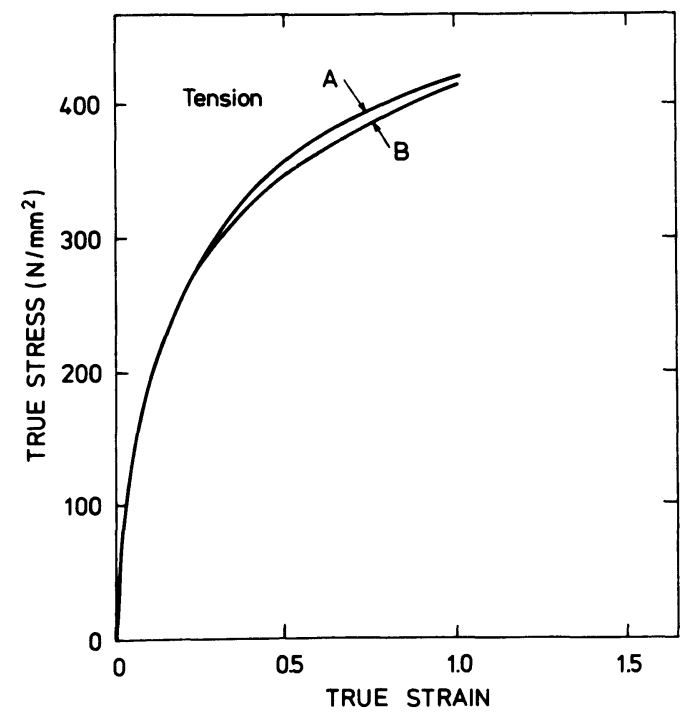

Fig. 11. True stress-true strain curves at room temperature of $99.999 \% \mathrm{Cu}$ in tension $\left(\dot{\varepsilon}=10^{-3}\right.$ $\mathrm{s}^{-1}$ ). The grain sizes were $23 \mu \mathrm{m}$ (curve marked A) and $125 \mu \mathrm{m}$ (curve marked B). From [43].

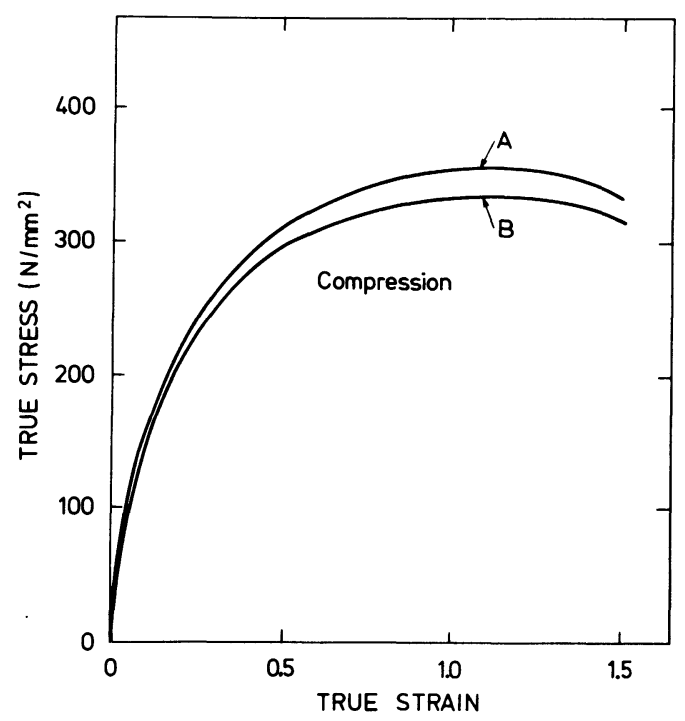

Fig. 12. True stress-true strain curves at room temperature for $99.999 \% \mathrm{Cu}$ in compression $(\dot{\varepsilon}=$ $10^{-3} \mathrm{~s}^{-1}$ ). The grain sizes were $23 \mu \mathrm{m}$ (curve marked A) and $125 \mu \mathrm{m}$ (curve marked B). From [43].

The flow stress-strain behaviour will in the following be related to observations of texture and microstructure.

5.2. Flow stress-texture (M factors).

The textural development during deformation (possibly in combination with a starting texture) will affect the flow stress-strain curve. This crystallographic effect can be analyzed by calculating the relevant $M$ factors on the basis of ODFs or inverse pole figures, usinr some selected deformation model. As an example figure 13 shows the relation between the $M$ factor and the plastic strain in tension. For comparison the $M$ factor derived on the basis of a simulated texture development is also shown. The Taylor model is used for both curves. Figure 13 illustrates that the $M$ factor variations are not very large $(5-10 \%)$, which is typical for cubic materials. The $M$ factor-strain relationship may be affected by metallurgical parameters but preliminary experiments $[34,43]$ related to the effect of grain size and of small particles indicate that this effect is not very large.

The relatively small variations in the M-factor with increasing strain shows that crystallographic strengthening can only account for a small fraction of the work hardening introduced during cold deformation. Furthermore the changes in the $M$ factor cannot account for the variations observed in the flow stress-strain relationship e.g. the transition from stage III to IV in tension or the flow softening observed in compression.

So far only those changes in $M$ which are produced directly by the change in crystallographic texture (with basically unchanged deformation mechanism) have been considered. M may also change as the 


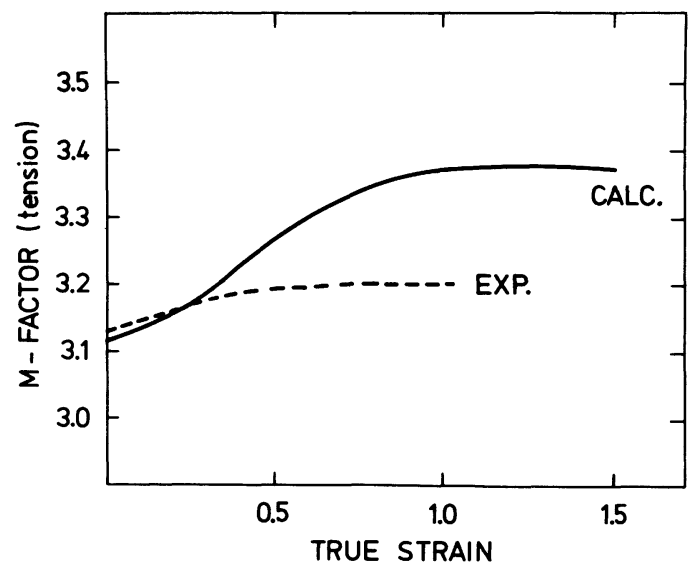

Fig. 13. Dependence of the Taylor factor $M$ on the true strain in tension. Calculations (replotted from [44]) are compared with results obtained on the basis of texture measurements for $99.999 \% \mathrm{Cu}$ with a grain size of $23 \mu \mathrm{m}$ [43].

result of a change in deformation mechanism (a change which may or may not be caused by the changes in crystallographic texture). The suggested change from full Taylor constraint to relaxed constraint when the shape of the grains changes (e.g. [44]) is one example of a change in mechanism which is accompanied by a change in $M$. This change has been suggested as the reason for work softening by compression [44]. However, such a change should also be reflected in a specific change in texture which was not observed experimentally in the copper case described above [43]. Another example of a change in deformation mechanism was considered by Hosford [45]: when a 〈110〉 fibre texture is developed (as in compressed f.c.c. materials), the deformation mode in the individual grains may change from axisymmetric to plane strain, the intergranular accommodation being accounted for by 'curling'. In the above case of compressed copper there was a reasonably strong $\langle 110\rangle$ texture, and grain curling was observed. The observed work softening may thus be the consequence of a change in $M$ because of a change to plane strain.

\subsection{Flow stress-microstructural evolution.}

It follows from the preceding subsection that the microstructural evolution must be considered in order to account for the observed changes in the flow stress as a function of strain. In general the microstructural development is characterised by an increase in the dislocation density. The flow stress $(\sigma)$ is related to the dislocation density $(p)$ by the general equation

$$
\sigma=a b G p^{1 / 2}
$$

where $\alpha$ is a constant (for which the magnitude may depend on the character of the dislocation arrangement), $b$ is the Burgers vector and $G$ is the shear modulus. At low and medium strains $p$ has been found to be proportional to the plastic strain [17]. However, at large strains the dependence of $p$ on strain is unknown except for the general observation that $\rho$ appears to increase with increasing strain. The observed increase in the flow stress with increasing strain is therefore in general accord with the observed changes in microstructure. The transition from stage III to stage IV has at present not been related to any microstructural observations, but one can list a number of specific features which do not appear to be the cause of this transition, e.g. [46]. It therefore seems likely that the explanation must be sought in the details of the microstructure e.g. In the rate of reduction of the cell size or in changes of the dislocation arrangements in the cell wall as the strain is increased.

In contrast to the general phenomenon of work hardening work softening is only characteristic for some metals and alloys and for certain deformation modes. It has been related to the occurrence of dynamic recovery, e.g. $[42,47]$. However, a more detailed explanation should be looked for, since in many systems, for instance pure aluminium, dynamic recovery does not give rise to work softening. The softening observed in compression of f.c.c. stuctures may be related to curling as discussed in 5.2 .

\subsection{Flow stress-cell size.}

The stress may be related to the microstructure through a relationship between the flow stress and some characteristic microstructural parameters. At present most of such studies have concentrated on the stress dependence of the cell size, but many results are inconclusive. This is for instance caused by the relatively large experimental scatter and the rather small variation in the cell size which normally can be obtained for a given set of experimental parameters. Furthermore the dislocation arrangements in the cell walls may depend on the experimental conditions, e.g. the deformation temperature. Thus, the experimental scatter may increase significantly when the analysis is based on a compilation of experiments obtained under different experimental conditions. The relation between the flow stress $(\sigma)$ and the cell size $(t)$ may be expressed by the equation

$$
\sigma-\sigma_{0}=K \cdot t^{-m}
$$

where $\sigma_{0}$ is a friction stress corresponding to very large $q$ values (zero strain) and $K$ and $m$ are constants. Normally $\mathrm{m}$ in equation (2) is taken to be either 0.5 or one. Equation (2) with $\mathrm{m}=0.5$ (and without friction stress) may be derived from equation (1) when it is assumed that the length of dislocation line per unit area of cell wall is constant. If the dislocation length per unit area increases with decreasing $t, m$ as derived from equation (1) will be bigger than 0.5 . In figure 14 data for cold drawn aluminium are plotted with $\mathrm{m}=$ 0.5 and $\mathrm{m}=1$. In both cases a linear plot is obtained. However, the intercept for very large values of $t$ is either too high $(m=1)$ or too low $(m=0.5)$ when compared with the yield stress of the undeformed material, which is $9 \mathrm{~N} / \mathrm{mm}^{2}$. Keeping this value fixed an $m$ value of approximately 0.8 gives the best fit. 


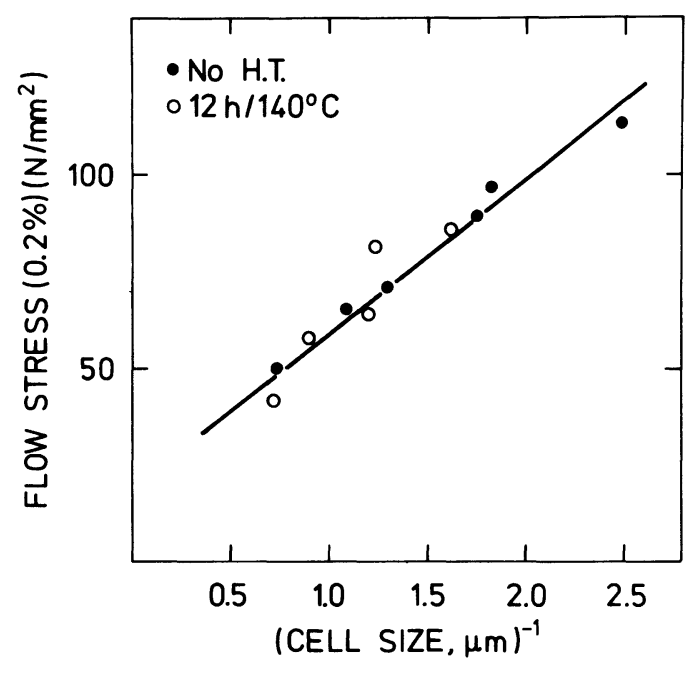

(a)

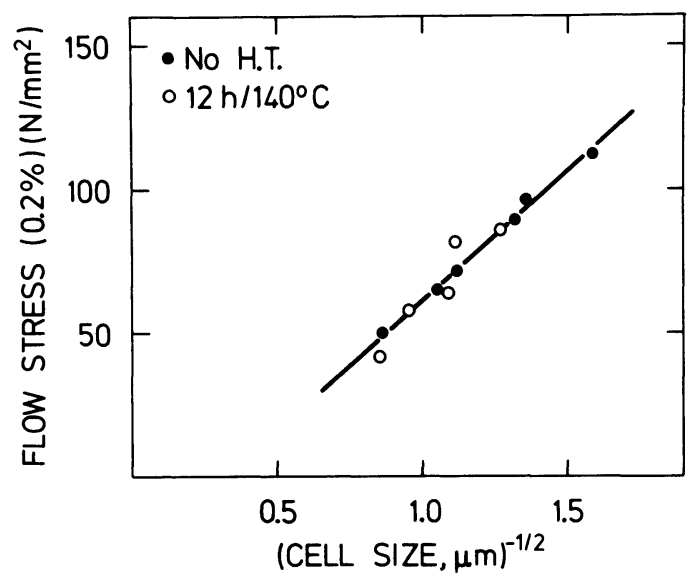

(b)

Fig. 14. Flow stress $(0.2 \%$ offset) at room temperature of $99.998 \% \mathrm{Al}$ as a function of the reciprocal cell size (a) and the reciprocal square root of the cell size (b).

Another version of equation (2) has been suggested [48] as the best fit for a very large number of different experiments:

$$
\frac{t}{b}=k\left(\frac{G}{\sigma}\right)^{m}
$$

where $\mathrm{K}$ and $\mathrm{m}$ are constants. This analysis showed very large variations in $m$ and $K$; for instance $m$ was found to vary in the range $0<m<2$ with a standard deviation of 0.39 . The large variation in $m$ and $K$ was explained in terms of random errors in the determination of the cell size as it was suggested that this parameter was known only within a factor of two. With this assumption $\mathrm{m}=0.84$ and $\mathrm{K}$ $=71$ produced the best fit, but the values $m=1$ and $\mathrm{K}=23$ also worked satisfactorily. However, for some materials (and experimental conditions) neither of these sets of values gave a good description.
The value 1 for $m$, expressing an inverse proportionality between the stress and the cell size, is in agreement with the theories of Kuhlmann-Wilsdorf $[49,50]$ and Holt $[51,52]$ both suggesting that this inverse proportionality can be accounted for on the basis of energy minimization during plastic flow. As concerns the value of $K$ this is also in rather good agreement with theoretical predictions [4952].

\subsection{Effect of metallurgical parameters.}

A change in the metallurgical parameters at medium and large strains normally leads to a shift in the numerical stress values whereas the shape of the stress-strain curve may be relatively unaffected. This has for instance been observed when the parameters are the grain size and the particle size and concentration, as will be discussed below.

a) Grain size. In tension (and in compression) a decrease in grain size shifts the stress-strain curve to a higher level. This grain size effect is well known at low and medium strain, but as shown in figure 11 the effect is also observed at large strains. Grain size strengthening may have its cause in an increase in the dislocation density at a given strain when the grain size is decreased. Such an increase has frequently been observed at medium strains, e.g. [17], whereas the structural information at high strains is scarce.

b) Particles. The introduction of particles into a metal leads to an increase in the flow stress, and a number of different strength contributions have been analyzed. These are the Orowan bowing stress, the mean stress and the forest hardening, the latter being caused by an increase in the dislocation density due to particles. The importance of these contributions depend on the plastic strain and the volume fraction of particles and their size and shape [53]. In alloys containing large particles the particle concentration is normally not very high and consequently particle strengthening is limited. In contrast, the presence of even a relatively small concentration of small particles may lead to significant strengthening. This is illustrated in figure 15 for two $\mathrm{Al}_{-} \mathrm{Al}_{2} \mathrm{O}_{3}$ materials. The figure shows that particle strengthening above a certain strain is additive to matrix strengthening. This observation is in agreement with the general finding [54] for dispersion strengthened materials that above a relatively small strain (approximately 0.05) work hardening is practically unaffected by the presence of particles. The large work hardening rate at small strains in particle containing materials has its cause in an increase in the dislocation density, whereas the strength-structure relationship at medium and large strains is not as clear. In this strain range an important effect of particles is to reduce the cell size at a given strain, and empirically it has been found that the flow stress-cell size relationship can be expressed by equation (2), where $\sigma_{0}$ now is the friction stress of the particle containing matrix. Alternative ways of adding the strength contributions have been discussed but in general the problem of additivity needs further 


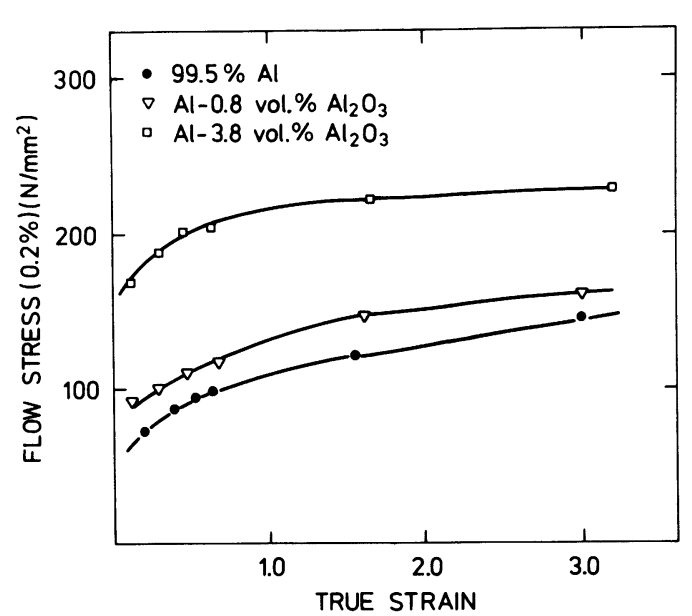

Fig. 15. Flow stress $(0.2 \%$ offset) as a function of strain by cold drawing of $99.5 \% \mathrm{Al}$

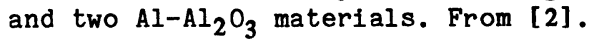

consideration - in parallel to the continued investigations of the individual strength contributions.

\subsection{Anisotropy.}

The crystallographic texture is the main cause of the anisotropy of the mechanical properties. How ever, microstructural contributions such as the distribution, shape and alignment of second phase particles, the shape of the grains and the alignment of dislocation boundaries, bands etc. may also be of importance. The effect of grain shape is basically the same as that considered earlier for texture and $M$ factor within the framework of the relaxed-constraint model. In practice there is the serious complication that stress and grain morphology are no longer aligned when one considers stresses different from those operating during the initial deformation of the material.

For materials with a crystallographic texture all elastic and plastic properties are anisotropic (apart from the elastic properties of materials with elastically isotropic grains). From a technological point of view the anisotropy of the formability is of great interest but here only the anisotropy of the flow stress will be considered. In general the anisotropy of the flow stress in cubic materials is not very great even at high strains.

In principle the models described in section 4 (and any similar model) can predict the relative directional variation in flow stress (and the shape of the yield surface) in materials with crystallographic texture. In practice the Taylor model (in the Bishop-Hill version [55]) has been used most extensively. The majority of the comparisons between theoretically derived and experimental anisotropy of the flow stress refer to annealed materials, whereas, in the present work, the interest is in deformed materials. The Bishop-Hill model seems to produce reasonably good but not perfect predictions of the anisotropy of the flow stress in deformed materials (e.g. [56]). However, because of the uncertainty about the structural contributions, such results should be interpreted with some caution.

\section{CONSTITUTIVE RELATIONS}

Constitutive equations or constitutive relations for the mechanical behaviour are of great importance in materials engineering. Those available today are largely on empirical basis, and they are mainly suited for interpolation in narrow parameter ranges. Constitutive relations with a better theoretical foundation could be used in a wider range of parameters. The development of such improved constitutive relations is therefore a matter of great concern.

A deformation model as described in section 4 is one of the elements in a theoretically based constitutive relation for the low-temperature mechanical behaviour at intermediate and large strain. If the model is realistic, it defines the stress state in the individual grains, the amount of shear on the different slip systems and the orientation of the slip systems. The other element is a relation between the shear pattern and the critical resolved shear stress, i.e. a microscopic workhardening law. An early attempt along this line was presented in [44], combining the relaxed-constraint model with an empirical isotropic law for hardening as a function of the sum of the shears.

The future effort to improve the constitutive relations will involve improvement of the models (as described in 4.3) combined with improvement of the microscopic work-hardening law. Ideally the hardening law should be based on a full understanding of the structural development and its relation to hardening. Alternatively one may use empirical hardening laws which include all the relevant parameters. Even the latter approach will require a substantial experimental and theoretical effort in order to identify the relevant parameters in the complex interaction between the dislocations belonging to the different slip systems. For practical applications it is important that the metallurgical parameters are among the parameters considered.

\section{CONCL UDING REMARKS}

Qualitatively the microstructural evolution with strain is relatively well known but quantitative correlations between the structural parameters and the strain has not yet been established. In this context, modern microscopical and non-microscopical techniques may offer new possibilities. Such techniques may also be useful in the characterization of individual structural features e.g. the dislocatioan arrangement in the cell walls.

The textural evolution as a function of strain is well characterized. However, modelling of this evolution shows discrepancies between experimental 
and calculated textures which should be resolved. This requires models which in more detail consider the nature of the slip proces including the plastic interaction between the grains.

The structural and textural information available at present provides the background for an understanding of the general stress-strain relationships even though a full understanding of various phenomena is still lacking. It has also been possible to establish empirical relations between the flow stress and some of the structural parameters, but more theoretical and experimental work is needed. A future goal will be to combine structural hardening and texture development in theoretically based constitutive relations.

\section{ACKNOWLEDGEMENTS}

The authors want to thank D. Juul Jensen and 0.B. Pedersen for helpful discussions and L. Jørgensen for typing the manuscript.

\section{REFERENCES}

[1] Low Energy Dislocation Structures, Eds. M.N. Bassim et al. (Elsevier Sequoia, Lausanne) 1986 .

[2] Hansen, N., Trans. Met. Soc. AIME 245 (1969) 2061.

[3] Kocks, U.F., Hasegawa, T. and Scattergood, R.O., Scr. Metall. 14 (1980) 449.

[4] Gil Sevillano, J., van Houtte, P. and Aernoudt, E., Prog. Mater. Sci. 25 (1980) 69.

[5] Swann, P.R., Electron Microscopy and Strength of Crystals, Eds. G. Thomas and J. Washburn (Wiley-Interscience, New York) 1963, p. 131.

[6] Young, C.T., Headley, T.J. and Lytton, J.L., Low Energy Dislocation Structures. Eds. M.N. Bassim et al. (Elsevier Sequoia, Lausanne) 1986 , p. 391 .

[7] Microstructural Characterization of Materials by Non-Microscopical Techniques, Eds. N. Hessel Andersen et al. (Risb National Laboratory, Roskilde) 1984

[8] Barlow, C.Y., Bay, B. and Hansen, N., Philos. Mag. 51 (1985) 253.

[9] Jackson, P.J., Scr. Metall. 17 (1983) 199.
[10] Nes, E., Hutchinson, W.B., Ridha A.A., Proc. 7th Int. Conf. on the Strength of Metals and Alloys, Eds. H.J. McQueen et al., (Pergamon, Oxford) 1985, p. 57.

[11] Koneva, N.A., Lychgin, D.V., Trishkina, L.T. and Kozlov, E.V., Proc. 7th Int. Conf. on the Strength of Metals and Alloys, Eds. H.J. McQueen et al. (Pergamon, Oxford) 1985, p. 21.

[12] Hansen, N. and Kuhlmann-Wilsdorf, D., Mat. Sci. Eng. 81 (1986) 141.

[13] Hansen, N. and Bay, B., Proc. 6th Int. Conf. on the Strength of Metals and Alloys, Ed. R.C. Gifkins (Pergamon, Oxford) 1982, p. 401.

[14] Malin, A.S. and Hatherly, M., Met. Sci. 13 (1979) 463.

[15] Korbel, A. and Martin, P., Acta Metall. 34 (1986) 1905

[16] Torrealdea, F.J. and Gil Sevillano, J., Proc. 6 th Int. Conf. on the Strength of Metals and Alloys, Ed. R.C. Gifkins (Pergamon, Oxford) 1982, p. 547.

[17] Hansen, N., Metall. Trans. A 16A (1985) 2167.

[18] Saimoto, S., Saka, H., Imura, T. and Yukawa, N., J. Electron. Micro. 26 (1977) 615.

[19] Hansen, N., Bay, B., Juul Jensen, D. and Leffers, T., Proc. 7th Int. Conf. on the Strength of Metals and Alloys, Ed. H.J. McQueen et al., (Pergamon Press, Oxford) 1985, p. 317.

[20] Humphreys, F.J., Acta Metall. 27 (1979) 1801.

[21] Humphreys, F.J., Textures and Microstructures 6 (1983) 45 .

[22] Bay, B. and Hansen, N., Metall. Trans. A 16A (1984) 287.

[23] Bay, B. and Hansen, N., Deformation of MultiPhase and Particle Containing Materials, Eds. J. Bilde-Sфrensen et al., (Risф National Laboratory, Roskilde) 1983, p. 145 .

[24] Sekine, K., Fukutomi, H. and Kamijo, T., Textures of Materials, Eds. C.M. Brakman et al. (Netherlands Society for Materials Science, Zwijndrecht) 1984 , p. 139 .

[25] Taylor, G.I., J. Inst. Met. 62 (1938) 307.

[26] Honeff, H. and Mecking, H., Textures of Materials, Eds. G. Gottstein and K. Lücke (Springer-Verlag, Berlin) 1978, p. 265.

[27] Kocks, U.F. and Canova, G.R., Deformation of Polycrystals: Mechanisms and Microstructures, Eds. N. Hansen et al., (Risф National Laboratory, Roskilde) 1981 , p. 35 .

[28] Leffers, T., Risф Report No. 184 (1968). 
[29] Leffers, T. and Juul Jensen, D., Textures and Microstructures 6 (1986) 231 .

[30] Grewen, J. and v.Heimendahl, M., Z. Metallkde. 59 (1968) 205.

[31] Duggan, B.J., Hatherly, M., Hutchinson, W.B. and Wakefield, P.T., Met. Sci. 12 (1978) 343.

[32] Leffers, T., Proc. ICOTOM 6, Ed. S. Nagashima (The Iron and Steel Institute of Japan, Tokyo) 1981 , p. 385 .

[33] Oztürk, T., Kallend, J.S. and Davies, G.J., Proc. ICOTOM 6, Ed. S. Nagashima (The Iron and Steel Institute of Japan, Tokyo) 1981, p. 507.

[34] Hansen, N. and Juul Jensen, D., Proc. Symp. on Formability and Metallurgical Structure (AIME) 1986.

[35] Leffers, T., Deformation of Polycrystals: Mechanisms and Microstructures, Eds. N. Hansen et al., (Risф National Laboratory, Roskilde) 1981 , p. 55 .

[36] Leffers, T., Scr. Metall. 9 (1975) 261.

[37] Berveiller, M., Hihi, A. and Zaoui, A., Deformation of Polycrystals: Mechanisms and Microstructures, Eds. N. Hansen et al. (Ris $\phi$ National Laboratory, Roskilde) 1981, p. 145.

[38] van Houtte, P., Proc. ICOTOM 6, Ed. S. Nagashima (The Iron and Steel Institute of Japan, Tokyo) 1981, p. 428.

[39] Kallend, J.S. and Davies, G.J., Philos. Mag. 25 (1972) 471.

[40] Asaro, R.J. and Needleman, A., Acta Metall. 33 (1985) 923 .

[41] Shuh, F. and v.Heimendahl, M., Z. Metallkde. 65 (1974) 346.
[42] Truckner, W.G. and Mikkola, D.E., Metall. Trans. A 8A (1977) 45.

[43] Naaman, H., Talreja, R., Juul Jensen, D. and Hansen, N., Textures and Microstructures (to be published).

[44] Tome, C., Canova, G.R., Kocks, U.F., Christodoulou, N. and Jonas, J.J., Acta Metall. 32 (1984) 1637.

[45] Hosford, W.F., Trans. Met. Soc. AIME 230 (1964) 12 .

[46] Hughes, D.A., Gibeling, J.C. and Nix, W.D., Proc. 7th Int. Conf. on the Strength of Metals and Alloys, Eds. H.J. McQueen et al. (Pergamon, Oxford) 1985 , p. 51

[47] Lloyd, D.J. and Kenny, D., Acta Metall. 28 (1980) 639.

[48] Ray, S.V. and Pharr, G.M., Low Energy Dislocation Structures, Eds. M.N. Bassim et al. (Elsevier Sequoia, Lausanne) 1986, p. 217.

[49] Kuhlmann-Wilsdorf, D., Metal]. Trans. 1 (1970) 3173 .

[50] Kuhlmann-Wilsdorf, D. and van der Merwe, J.H., Mater. Sci. Eng. 55 (1982) 79.

[51] Holt, D.L., J. Appl. Phys. 41 (1970) 3197.

[52] Staker, M.R. and Holt, D.L., Acta Metall. 20 (1972) 569.

[53] Deformation of Multi-Phase and Particle Containing Materials, Eds. J. Bilde-Sфrensen et al., (Risb National Laboratory, Roskilde) 1983.

[54] Kelly, A. and Nicholson, R.B., Progr. Mat. Sci. 10 (1963) 151.

[55] Bishop, J.F.W. and Hill, R., Philos. Mag. 42 (1951) 414 and 1298

[56] Kallend, J.S. and Davies, G.J., J. Inst. Met. 5 (1971) 257. 